\title{
Quark contribution to the proton spin from 2+1+1-flavor lattice QCD
}

\author{
Huey-Wen Lin, ${ }^{1,2, *}$ Rajan Gupta, ${ }^{3, \dagger}$ Boram Yoon, ${ }^{3, \ddagger}$ Yong-Chull Jang, ${ }^{4, \S}$ and Tanmoy Bhattacharya ${ }^{3, \|}$ \\ (PNDME Collaboration) \\ ${ }^{1}$ Department of Physics and Astronomy, Michigan State University, East Lansing, Michigan 48824, USA \\ ${ }^{2}$ Department of Computational Mathematics, Science and Engineering, Michigan State University, \\ East Lansing, Michigan 48824, USA \\ ${ }^{3}$ Los Alamos National Laboratory, Theoretical Division T-2, Los Alamos, New Mexico 87545, USA \\ ${ }^{4}$ Brookhaven National Laboratory, Physics Department, Upton, New York 87545, USA
}

(Received 28 June 2018; published 30 November 2018)

\begin{abstract}
We present the first chiral-continuum extrapolated up, down, and strange quark spin contribution to the proton spin using lattice QCD. For the connected contributions, we use 11 ensembles of $2+1+1$-flavor of highly improved staggered quarks (HISQ) generated by the MILC Collaboration. They cover four lattice spacings, $a \approx\{0.15,0.12,0.09,0.06\} \mathrm{fm}$, and three pion masses, $M_{\pi} \approx\{315,220,135\} \mathrm{MeV}$, of which two are at the physical pion mass. The disconnected strange calculations are done on seven of these ensembles, covering the four lattice spacings but only one with the physical pion mass. The disconnected light quark calculation was done on six ensembles at two values of $M_{\pi} \approx\{315,220\} \mathrm{MeV}$. High-statistics estimates on each ensemble for all three quantities allow us to quantify systematic uncertainties and perform a simultaneous chiral-continuum extrapolation in the lattice spacing and the light-quark mass. Our final results are $\Delta u \equiv\langle 1\rangle_{\Delta u^{+}}=0.777(25)(30), \Delta d \equiv\langle 1\rangle_{\Delta d^{+}}=-0.438(18)(30)$, and $\Delta s \equiv\langle 1\rangle_{\Delta s^{+}}=-0.053(8)$, adding up to a total quark contribution to proton spin of $\sum_{q=u, d, s}\left(\frac{1}{2} \Delta q\right)=0.143(31)(36)$. The second error is the systematic uncertainty associated with the chiral-continuum extrapolation. These results are obtained without model assumptions and are in good agreement with the recent COMPASS analysis $0.13<\frac{1}{2} \Delta \Sigma<0.18$ and with the $\Delta q$ obtained from various global analyses of polarized beam or target data.
\end{abstract}

DOI: 10.1103/PhysRevD.98.094512

\section{INTRODUCTION}

In 1987, the European Muon Collaboration measured the spin asymmetry in polarized deep inelastic scattering and presented the remarkable result that the sum of the spins of the quarks contributes less than half of the total spin of the proton [1]. This unexpected result was termed the "proton spin crisis." Lattice QCD can unravel the mystery of where the proton gets its spin by measuring the matrix elements of appropriate quark and gluon operators within the nucleon state. In this paper, we present the first lattice calculation of the contribution of the intrinsic spin of the quarks to the

\footnotetext{
*hwlin@pa.msu.edu

†rajan@lanl.gov

tboram@lanl.gov

§yj@bnl.gov

"tanmoy@lanl.gov
}

Published by the American Physical Society under the terms of the Creative Commons Attribution 4.0 International license. Further distribution of this work must maintain attribution to the author(s) and the published article's title, journal citation, and DOI. Funded by SCOAP. proton spin with high-statistics and control over systematic errors. Our result, $\sum_{q=u, d, s} \frac{1}{2} \Delta q=0.143(31)(36)$, is in good agreement with the COMPASS analysis $0.13<$ $\frac{1}{2} \Delta \Sigma<0.18$ at $3 \mathrm{GeV}^{2}$ [2]. Note that, above $3 \mathrm{GeV}^{2}$, the change of the axial charges with scale is negligible.

To calculate the nucleon spin using lattice QCD, one starts with Ji's sum rule [3] that provides a gauge invariant decomposition of the nucleon's total spin as

$$
\frac{1}{2}=\sum_{q=u, d, s, c, .}\left(\frac{1}{2} \Delta q+L_{q}\right)+J_{g}
$$

where $\Delta q \equiv \Delta \Sigma_{q} \equiv\langle 1\rangle_{\Delta q^{+}} \equiv g_{A}^{q}$ is the contribution of the intrinsic spin of a quark with flavor $q ; L_{q}$ is the orbital angular momentum of that quark; and $J_{g}$ is the total angular momentum of the gluons. Thus, to explain the spin of the proton starting from QCD, one needs to calculate the contributions of all three terms. In this paper we present results for the relatively better determined first term, $\frac{1}{2} \Delta \Sigma \equiv \sum_{q=u, d, s} \frac{1}{2} \Delta q$.

On the lattice, the axial charge $g_{A}^{q}$ is given by the matrix element of the flavor diagonal axial current, $\bar{q} \gamma_{\mu} \gamma_{5} q$, 


$$
g_{A}^{q} \bar{u}_{N} \gamma_{\mu} \gamma_{5} u_{N}=\left\langle N\left|Z_{A} \bar{q} \gamma_{\mu} \gamma_{5} q\right| N\right\rangle,
$$

where $Z_{A}$ is the renormalization constant and $u_{N}$ is the neutron spinor. In addition to quantifying the contribution of the quarks to the nucleon spin,

$$
g_{A}^{q} \equiv \Delta q=\int_{0}^{1} d x(\Delta q(x)+\Delta \bar{q}(x))
$$

is also the first Mellin moment of the polarized parton distribution function (PDF) integrated over the momentum fraction $x$ [4]. The charges, $g_{A}^{u, d, s}$, also quantify the strength of the spin-dependent interaction of dark matter with nucleons [5,6]. Of these, $\Delta s$ is the least well known and current analyses [4] often rely on assumptions such as $\mathrm{SU}(3)$ symmetry and $\Delta s=\Delta \bar{s}$.

\section{LATTICE METHODOLOGY}

The calculation of the flavor diagonal charges $g_{A}^{q}$ is now mature $[7,8]$. The challenge is to obtain high-statistics results for both the connected and disconnected contributions to nucleon three-point functions illustrated in Fig. 1 and then address the various systematics. An important finding of this work is that the lattice discretization errors and the chiral corrections are large; consequently, the evaluation of the renormalized charges at the physical pion mass, $M_{\pi^{0}}=135 \mathrm{MeV}$, and the extrapolation to the continuum limit are essential, as discussed in Sec. V.

The calculations of the connected and disconnected contributions to $g_{A}^{u, d}$ were done separately using $2+1+1$-flavor ensembles of HISQ fermions [9] generated by the MILC Collaboration [10]. The construction of the two- and three-point correlation functions used in the analysis was carried out using Wilson-clover fermions. We refer to this as the clover-on-HISQ lattice formulation, which in the continuum limit is expected to give results for QCD. All results presented here are for degenerate $u$ and $d$ quarks, with the $s$ and $c$ quark masses tuned to their physical values.

Results for the connected contributions have been obtained using 11 HISQ ensembles that cover the range $0.06 \lesssim a \lesssim 0.15 \mathrm{fm}$ in the lattice spacing, $135 \lesssim M_{\pi} \lesssim$ $320 \mathrm{MeV}$ in the pion mass, and $3.3 \lesssim M_{\pi} L \lesssim 5.5$ in the
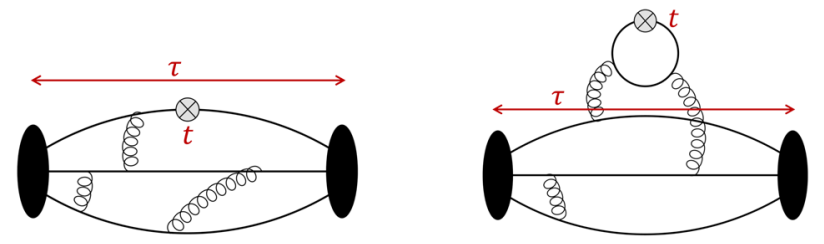

FIG. 1. The connected (left) and disconnected (right) threepoint diagrams that contribute to the flavor diagonal matrix elements of the axial operator (labeled by $\otimes$ at time slice $t$ ) within the nucleon state. The black blobs denote nucleon source and sink, separated by Euclidean time $\tau$. spatial lattice size expressed in terms of $M_{\pi} L$. The analysis of the connected contributions, including the simultaneous chiral-continuum-finite-volume (CCFV) fits, has been presented in Ref. [8], and the final results are

$$
\begin{aligned}
g_{A}^{u-d} & =1.218(25)(30), \\
\left.g_{A}^{u}\right|_{\mathrm{conn}} & =0.895(21), \\
\left.g_{A}^{d}\right|_{\text {conn }} & =-0.320(12) .
\end{aligned}
$$

The second error in $g_{A}^{u-d}$ represents an estimate of the uncertainty due to using the leading order corrections in the CCFV fit ansatz.

The computationally expensive calculations of the disconnected contributions has been carried out on six (for light $u$ and $d$ quark contributions) and seven (for strange quark) HISQ ensembles, as described in Table I. The calculation of the vacuum polarization loop with the current insertion in the disconnected diagram is carried out stochastically using Gaussian or $Z_{4}$ random sources on each background gauge configuration, as described in Ref. [7]. In this method, the final statistical error is a combination of the error in the stochastic evaluation on each configuration and the error due to the average over the gauge configurations required by the path integral.

To increase the statistics in a cost-effective manner, the calculations of both the two- and three-point nucleon correlation functions were carried out using the truncated solver method with bias correction $[11,12]$. In this method, correlation functions are constructed using quark propagators inverted with low precision (LP) stopping criteria between $r_{\mathrm{LP}} \equiv \mid$ residue $\left.\right|_{\mathrm{LP}} / \mid$ source $\mid=10^{-3}$ and $5 \times 10^{-4}$ and high precision (HP) with $r_{\mathrm{HP}}$ between $10^{-7}$ and $10^{-8}$ $[7,8]$. The bias corrected correlation functions on each configuration are given by

$$
C^{\mathrm{imp}}=\sum_{i=1}^{N_{\mathrm{LP}}} \frac{C_{\mathrm{LP}}\left(\mathbf{x}_{i}^{\mathrm{LP}}\right)}{N_{\mathrm{LP}}}+\sum_{i=1}^{N_{\mathrm{HP}}}\left[\frac{C_{\mathrm{HP}}\left(\mathbf{x}_{i}^{\mathrm{HP}}\right)-C_{\mathrm{LP}}\left(\mathbf{x}_{i}^{\mathrm{HP}}\right)}{N_{\mathrm{HP}}}\right],
$$

where $C_{\mathrm{LP}}$ and $C_{\mathrm{HP}}$ are the two- or three-point functions calculated in LP and HP, respectively, and $\mathbf{x}_{i}^{\mathrm{LP}}$ and $\mathbf{x}_{i}^{\mathrm{HP}}$ are the source positions for the two kinds of propagator inversion. The bias was found to be smaller than the statistical errors in all cases.

\section{EXCITED-STATE CONTAMINATION}

To obtain the nucleon charges, we need to evaluate the matrix elements of the corresponding quark bilinear operators within the ground state of the nucleons. We use the same toolkit to remove the excited-state contamination (ESC) that is described in Refs. [7,8]: three-state (two-state) fits to data for the connected (disconnected) three-point functions as a function of both the operator insertion time $t$ and multiple source-sink separation $\tau$. The overlap with the 
TABLE I. Lattice parameters of the seven ensembles analyzed for the disconnected contributions. This table gives the number of configurations analyzed for the light $\left(N_{\text {conf }}^{l}\right)$ and strange $\left(N_{\text {conf }}^{s}\right)$ quarks, the number of random sources $\left(N_{\text {src }}\right)$, and the ratio $N_{\mathrm{LP}} / N_{\mathrm{HP}}$ of LP to HP solves used to estimate the quark loop on each configuration. The parameters of the 11 ensembles used for the connected contribution are given in Table 1 of Ref. [8].

\begin{tabular}{lccccccccc}
\hline \hline Ensemble ID & $a(\mathrm{fm})$ & $M_{\pi}(\mathrm{MeV})$ & $L^{3} \times T$ & $M_{\pi} L$ & \multicolumn{1}{c}{$N_{\text {conf }}^{l}$} & \multicolumn{1}{c}{$N_{\text {src }}^{l}$} & \multicolumn{1}{c}{$N_{\text {conf }}^{s}$} & \multicolumn{1}{c}{$N_{\text {src }}^{s}$} & $N_{\mathrm{LP}} / N_{\mathrm{HP}}$ \\
\hline$a 15 m 310$ & $0.1510(20)$ & $320(5)$ & $16^{3} \times 48$ & 3.93 & 1917 & 2000 & 1919 & 2000 & 50 \\
$a 12 m 310$ & $0.1207(11)$ & $310(3)$ & $24^{3} \times 64$ & 4.55 & 1013 & 5000 & 1013 & 1500 & 30 \\
$a 12 m 220$ & $0.1184(10)$ & $228(2)$ & $32^{3} \times 64$ & 4.38 & 958 & 11000 & 958 & 4000 & 30 \\
$a 09 m 310$ & $0.0888(08)$ & $313(3)$ & $32^{3} \times 96$ & 4.51 & 1081 & 4000 & 1081 & 2000 & 30 \\
$a 09 m 220$ & $0.0872(07)$ & $226(2)$ & $48^{3} \times 96$ & 4.79 & 712 & 8000 & 847 & 10000 & $30 / 50$ \\
$a 09 m 130$ & $0.0871(06)$ & $138(1)$ & $64^{3} \times 96$ & 3.90 & & & 877 & 10000 & 50 \\
$a 06 m 310$ & $0.0582(04)$ & $320(2)$ & $48^{3} \times 144$ & 4.52 & 830 & 4000 & $200+340$ & $5000+10000$ & 50 \\
\hline \hline
\end{tabular}

ground state is increased by using Gaussian smeared sources for propagator calculation. The root-mean-square smearing radius is tuned to be between 0.6 and $0.7 \mathrm{fm}$. All correlation functions are constructed using these propagators smeared at the source and the sink. The masses and amplitudes of the states are extracted from the two-point functions constructed using four-state fits.
The details of these analyses have already been published in Ref. [8].

The data and the two-state fits for the disconnected contributions are shown in Fig. 2. The data are noisier compared to the connected part analyzed in Ref. [8]. Because of the weaker statistical signal, the two-state fits to the three-point function are, in some cases, more
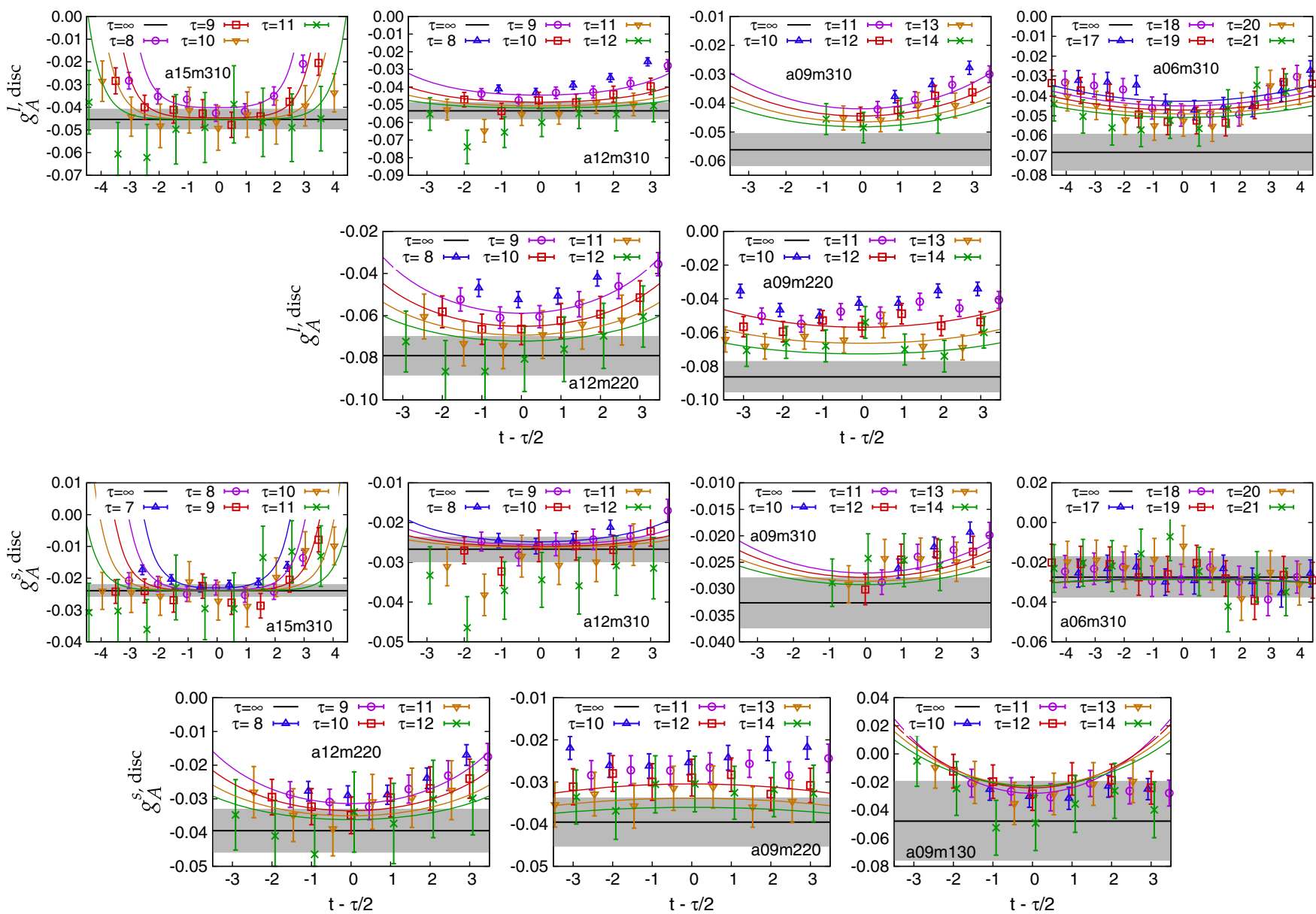

FIG. 2. The data and the two-state fit to the light (top two rows) and strange (bottom two rows) quark disconnected contribution to the bare $g_{A}^{(l, s) \text {,disc }}$. The grey error band and the solid line within it is the $\tau \rightarrow \infty$ estimate obtained using the two-state fit to data at different $t$ and $\tau$. The result of the fit for each individual $\tau$ is shown by a solid line in the same color as the data points. 
weighted towards smaller values of the source-sink separation $\tau$. Also, in many cases there is no clear pattern of convergence towards the $\tau \rightarrow \infty$ value. We, therefore, first determined the direction of convergence versus $\tau$ for both $g_{A}^{l}$ and $g_{A}^{s}$ by analyzing data at small $\tau$ that have smaller statistical errors but larger ESC. We then take the largest range of $\tau$, shown in Fig. 2, for which the errors are reasonable and the entries in the covariance matrix used in the two-state fits are stable under variation in the set of values of $t$ and $\tau$ used. Because of the difference in the quality of the statistical signal, and because the number of ensembles and configurations analyzed are not the same, we carry out separate analyses of the connected and disconnected contributions.

Analyzing the connected and disconnected contributions separately to remove ESC introduces an approximation. To define connected and disconnected contributions individually, one has to work in a partially quenched theory with an additional quark with flavor $u^{\prime}$. However, in this theory, the Pauli exclusion principle does not apply between the $u$ and $u^{\prime}$ quarks. The upshot of this is that the spectrum of states in the partially quenched theory is larger; for example, an intermediate $u^{\prime} u d$ state would be the analogue of a $\Lambda$ rather than a nucleon [13]. Thus, the spectral decomposition for this partially quenched theory and QCD is different. In the ESC fits, we however use the same QCD spectral decomposition in the fits for both the two- and three-point functions, whereas one should be using the partially quenched spectrum for the three-point function. The size of the extrapolation under consideration is the difference between the value at $t=\tau / 2$ for the largest $\tau$ and the asymptotic value, whose estimate is the grey band. Since this difference should converge exponentially as $\tau \rightarrow \infty$ and is observed to be small ( $<0.02)$, as shown in Fig. 2, we assume that any additional systematic in the extrapolation due to not using the partially quenched spectrum is well within the quoted uncertainty. We have also found that in the fits to both connected and disconnected contributions, the extrapolated value is not very sensitive to the precise values of the amplitudes and masses used, i.e., whether they are taken from three- or four-state fits to the two-point functions.

In the fits to remove ESC, we underscore the observation that the disconnected contribution converges from above, while the connected contribution converges from below as shown in Ref. [8]; i.e., the ESC in the disconnected threepoint function is opposite to that observed in the connected three-point function. In both cases, removing the ESC increases the magnitude of their contribution, with the disconnected contribution becoming more negative. Note that such an increase in the negative contribution from the sea quarks reduces the fraction of the nucleon spin carried by the quarks.

The final results for the bare values of the light $(u, d)$, $g_{A}^{l, \text { bare }}$, and strange quarks, $g_{A}^{s, \text { bare }}$, obtained from the twostate fits are collected together in Table II.

\section{RENORMALIZATION OF THE OPERATORS}

The renormalization of flavor diagonal light quark operators, $\bar{q} \gamma_{\mu} \gamma_{5} q$, requires knowing both nonsinglet and singlet factors [14]. In this work, we neglect the difference between the two and renormalize all charges using $Z_{A}^{\text {isovector }}$ calculated in the regularization-independent symmetric momentum-subtraction (RI-sMOM) scheme and converted to the MS scheme at $2 \mathrm{GeV}$ using two-loop perturbation theory. These results are given in Ref. [8]. In perturbation theory, the difference between the two starts at two loops is shown in Ref. [15]; however, the numerical value is small, $O(0.01)$. Explicit nonperturbative calculations find that $Z_{A}^{\text {nonsinglet }}$ and $Z_{A}^{\text {singlet }}$ agree to within a percent for the twisted mass and the clover-Wilson actions [16-18]. While we have not checked that the difference is similarly small also for our clover-on-HISQ calculation, we assume it is

TABLE II. The bare and renormalized charges from the different ensembles are given along with the values after extrapolation to $a=0$ and $M_{\pi}=135 \mathrm{MeV}$. The charges, renormalized at $2 \mathrm{GeV}$ in the $\overline{\mathrm{MS}}$ scheme in the two ways defined in Eq. (5), are given in columns 4-7. In all cases, the numbers within the square brackets are the $\chi^{2} /$ DOF of the fits. In the ESC fits for extracting the bare charges, shown in Fig. 2, the $\chi^{2} /$ DOF with DOF $\approx 20$ is given in columns $2-3$. In the chiral-continuum fits, using Eq. (6) and shown in Fig. 3, the $\chi^{2} / \mathrm{DOF}$ with $\mathrm{DOF}=3$ (light) or 4 (strange) is given in the last row.

\begin{tabular}{|c|c|c|c|c|c|c|}
\hline ID & $g_{A}^{l, \text { bare }}$ & $g_{A}^{s, \text { bare }}$ & $\left.g_{A}^{l}\right|_{R 1}$ & $\left.g_{A}^{s}\right|_{R 1}$ & $\left.g_{A}^{l}\right|_{R 2}$ & $\left.g_{A}^{s}\right|_{R 2}$ \\
\hline $\mathrm{a} 15 \mathrm{~m} 310$ & $-0.045(4)[0.9]$ & $-0.024(2)[1.2]$ & $-0.044(4)$ & $-0.023(2)$ & $-0.045(4)$ & $-0.024(2)$ \\
\hline $\begin{array}{l}\text { a } 12 \mathrm{~m} 310 \\
\mathrm{a} 12 \mathrm{~m} 220\end{array}$ & $\begin{array}{l}-0.053(5)[1.2] \\
-0.079(9)[0.8]\end{array}$ & $\begin{array}{l}-0.027(3)[1.1] \\
-0.039(6)[0.7]\end{array}$ & $\begin{array}{l}-0.051(5) \\
-0.075(9)\end{array}$ & $\begin{array}{l}-0.025(3) \\
-0.037(6)\end{array}$ & $\begin{array}{l}-0.052(4) \\
-0.077(9)\end{array}$ & $\begin{array}{l}-0.026(3) \\
-0.038(6)\end{array}$ \\
\hline $\begin{array}{l}\mathrm{a} 09 \mathrm{~m} 310 \\
\mathrm{a} 09 \mathrm{~m} 220 \\
\mathrm{a} 09 \mathrm{~m} 130\end{array}$ & $\begin{array}{l}-0.056(6)[0.8] \\
-0.086(9)[1.3]\end{array}$ & $\begin{array}{l}-0.033(5)[0.9] \\
-0.040(6)[1.3] \\
-0.048(28)[1.3]\end{array}$ & $\begin{array}{l}-0.053(6) \\
-0.082(9)\end{array}$ & $\begin{array}{l}-0.031(5) \\
-0.038(6) \\
-0.046(27)\end{array}$ & $\begin{array}{l}-0.056(6) \\
-0.085(9)\end{array}$ & $\begin{array}{l}-0.033(5) \\
-0.039(6) \\
-0.047(27)\end{array}$ \\
\hline $\mathrm{a} 06 \mathrm{~m} 310$ & $-0.068(9)[0.8]$ & $-0.027(10)[1.3]$ & $-0.066(9)$ & $-0.026(10)$ & $-0.068(9)$ & $-0.027(10)$ \\
\hline Extrapolated & & & $-0.115(13)[0.28]$ & $-0.052(8)[0.17]$ & $-0.120(14)[0.20]$ & $-0.054(8)[0.21]$ \\
\hline
\end{tabular}


covered by the $O(0.03)$ uncertainty in the calculated values of $Z_{A}^{\text {isovector }} \equiv Z_{A}^{u-d}$ used. In short, both the disconnected and connected contributions are renormalized in the following two ways:

$$
\begin{aligned}
& \left.g_{A}\right|_{R 1}=g_{A} \times Z_{A}^{\text {isovector }}, \\
& \left.g_{A}\right|_{R 2}=\frac{g_{A}}{g_{V}^{u-d}} \times \frac{Z_{A}^{\text {isovector }}}{Z_{V}^{u-d}},
\end{aligned}
$$

with the values of $Z_{A}^{\text {isovector }}$ and $Z_{V}^{u-d}$ taken from Ref. [8]. In the second method, the conserved vector current relation $g_{V}^{u-d} \times Z_{V}^{u-d}=1$ is implicit. The final values are taken to be the average of the two after performing the chiral-continuum extrapolation. The results for disconnected contributions are given in Table II and the connected contributions, taken from Ref. [8], are reproduced in Eq. (4).

\section{THE CONTINUUM-CHIRAL EXTRAPOLATION}

The leading discretization effects are taken to be linear in $a$ since the action and the operators in our clover-on-HISQ formalism are not fully $O(a)$ improved. We take the leading dependence on $M_{\pi}$ from the finite volume chiral perturbation theory [19-25], which is proportional to $M_{\pi}^{2}$. We neglect finite volume corrections since no significant evidence for them was found in the dominant connected contributions [8]. In Fig. 3, we show the simultaneous chiral-continuum fits (pink band) versus $a$ and $M_{\pi}^{2}$ to the renormalized disconnected data $g_{A}^{l, s}$ given in Table II using the ansatz,

$$
g_{A}^{l, s}\left(a, M_{\pi}, L\right)=c_{1}+c_{2} a+c_{3} M_{\pi}^{2} .
$$
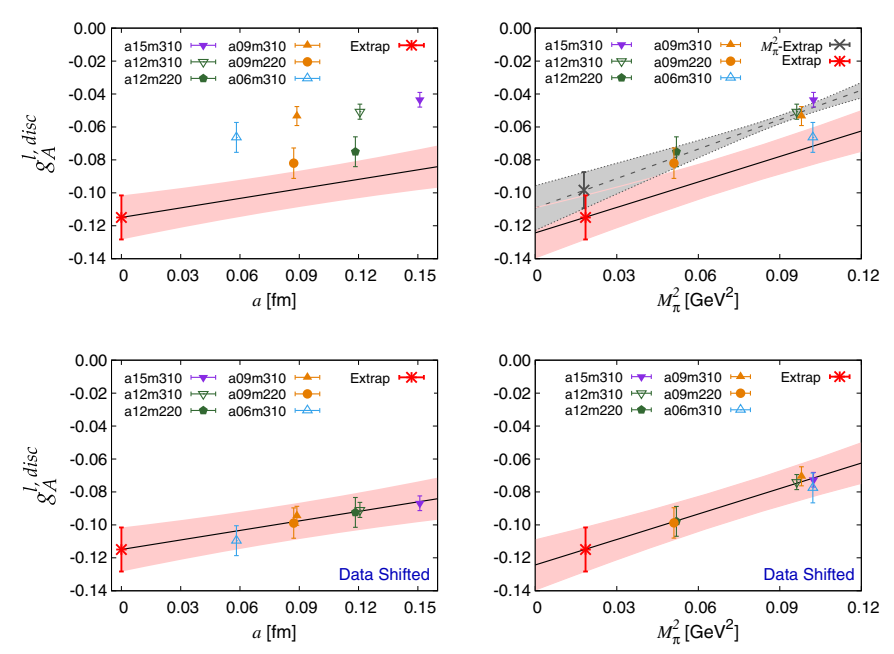

The results of the extrapolated values from the fits for both renormalization procedures are also given in Table II along with the $\chi^{2} / \mathrm{DOF}$. For comparison, the grey band within dotted lines in Fig. 3 is the fit to a single variable $M_{\pi}^{2}$, i.e., with $c_{2}=0$. The difference between the two bands highlights the need for the simultaneous fit.

The results for the fit parameters $c_{i}$ for the light and strange quarks are given in Table III. From the fits shown in Fig. 3, it is clear that, even with the limited number of data points, the signal is good enough to give a statistically significant determination of the $c_{i}$, and show that the variation is essentially linear in the two variables. The change in $c_{i}$ going from the light to the strange quark is also clear from the data and the fits.

We also carried out fits including the next order corrections, $a^{2}$ for the discretization errors and $M_{\pi}^{2} \log M_{\pi}^{2}$ for the chiral $\log$ term, one at a time. In each case, the errors in the coefficients and in the results grow. For example, in the best case of adding the $a^{2}$ term as there are data at four values of $a$, we get $\left.g_{A}^{l}\right|_{R 2}=-0.147(43)$, and the coefficients $c_{2}=0.67(71)$ and $c_{a^{2}}=-2.1(3.2)$. The $\chi^{2}=0.6$ of the fit using Eq. (6), which was already unreasonably small, decreased to 0.3 . There was no scope to reduce $\chi^{2}$ by two units as is required by the Akaike information criteria [26] to warrant including additional terms. In fact, as is obvious, such statistical tests are meaningless for such small $\chi^{2}$ values. More importantly, within the range of the data, $c_{2}$ and $c_{a^{2}}$ compete to reduce $\chi^{2}$ but both are poorly determined. Outside, the predictive power of the fit deteriorates as is typical of overparametrized fits. Our conclusion is that the ansatz given in Eq. (6) is sufficient to fit the current data and many more data
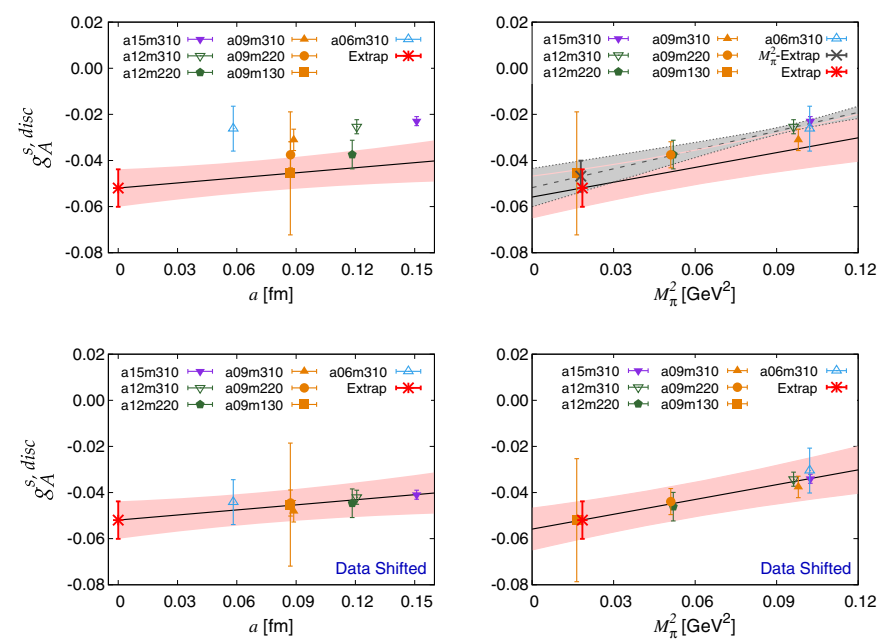

FIG. 3. Top: The extrapolation of the renormalized $g_{A}^{l \text {,disc }}$ and $g_{A}^{s, \text { disc }}$ data using the chiral-continuum ansatz given in Eq. (6). In each panel, the pink band shows the result of the simultaneous fit plotted versus a single variable with the other variable set to its physical value. The result at the physical point, $M_{\pi}=135 \mathrm{MeV}$ and $a=0$, is marked with a red star. The grey band shows the fit versus only $M_{\pi}$, i.e., ignoring the dependence on $a$. It highlights the need for a simultaneous fit in both $a$ and $M_{\pi}$. Bottom: The data in each panel are plotted after extrapolation to the physical point ( $a=0$ or $M_{\pi}=135 \mathrm{MeV}$ ) in the nonplotted variable to facilitate comparison with the simultaneous fit. 
TABLE III. The values of the parameters $c_{i}$, defined in Eq. (6), for the final fits shown in Fig. 3 and the results given in Table II.

\begin{tabular}{lcccc}
\hline \hline & $\left.g_{A}^{l}\right|_{R 1}$ & \multicolumn{1}{c}{$\left.g_{A}^{s}\right|_{R 1}$} & \multicolumn{1}{c}{$\left.g_{A}^{l}\right|_{R 2}$} & $\left.g_{A}^{s}\right|_{R 2}$ \\
\hline$c_{1}$ & $-0.124(15)$ & $-0.056(9)$ & $-0.129(15)$ & $-0.058(9)$ \\
$c_{2}\left(\mathrm{fm}^{-1}\right)$ & $0.193(89)$ & $0.073(65)$ & $0.207(88)$ & $0.084(65)$ \\
$c_{3}\left(\mathrm{GeV}^{-2}\right)$ & $0.52(15)$ & $0.21(10)$ & $0.53(15)$ & $0.22(10)$ \\
$\chi^{2} / \mathrm{DOF}$ & 0.281 & 0.167 & 0.203 & 0.205 \\
\hline \hline
\end{tabular}

TABLE IV. Our results for the $u, d$, and $s$ quarks, after extrapolation to $a=0$ and $M_{\pi}=135 \mathrm{MeV}$, for the connected and disconnected contributions and their sum are given in the first three rows. The sum over flavors gives $\Delta \Sigma=\Delta u+\Delta d+\Delta s=$ $0.286(62)$. ETMC results at the single lattice spacing $a=$ $0.0938 \mathrm{fm}$ [17] are given in the last row.

\begin{tabular}{lcll}
\hline \hline & \multicolumn{1}{c}{$g_{A}^{u} \equiv \Delta u$} & \multicolumn{1}{c}{$g_{A}^{d} \equiv \Delta d$} & \multicolumn{1}{c}{$g_{A}^{s} \equiv \Delta s$} \\
\hline Connected & $0.895(21)(30)$ & $-0.320(12)(30)$ & \\
Disconnected & $-0.118(14)$ & $-0.118(14)$ & $-0.053(8)$ \\
Sum & $0.777(25)(30)$ & $-0.438(18)(30)$ & $-0.053(8)$ \\
ETMC & $0.830(26)$ & $-0.386(18)$ & $-0.042(10)(2)$ \\
\hline \hline
\end{tabular}

points are needed to explore additional corrections. Unfortunately, the analysis of the remaining HISQ ensembles at smaller $a$, and those at the physical pion mass, has not yet been possible due to the computational cost. Our final results are, therefore, derived from fits using Eq. (6). To account for the uncertainty in the fit model, we assign an additional systematic error of 0.03 , coming from the connected contributions, in $g_{A}^{u}$ and $g_{A}^{d}$.

Results for the individual contributions are collected together in Table IV, along with the connected contributions reproduced from Ref. [8]. Their sum $\frac{1}{2} \Delta \Sigma \equiv$ $\sum_{q=u, d, s} \frac{1}{2} \Delta q=0.143(31)$ is in good agreement with the COMPASS result [2]. Scaling our value of $g_{A}^{s}$ by $1 / m_{q}$ suggests that the neglected charm contribution could be $g_{A}^{c} \approx-0.005$.

Our result $g_{A}^{u-d}=1.218(27)(30)$ for the isovector axial charge is 0.058 below the experimental value
$g_{A}^{u-d}=1.2766(20)$, as discussed in Ref. [8]. The difference can be explained if the connected $g_{A}^{u}$ is underestimated by 0.058 or $g_{A}^{d}$ is more negative by this amount or any combination of the two. (The disconnected contributions cancel in $g_{A}^{u-d}$.) In the first case, $\Delta \Sigma / 2$ would increase by 0.029 , and in the second case decrease by the same amount. The most likely reason for this underestimate is the uncertainty in the chiral-continuum-finite-volume extrapolation, which was estimated to be 0.03 in Ref. [8], independent of the experimental value. This systematic uncertainty has been added as a second error in $g_{A}^{u}$ and $g_{A}^{d}$. In the fits to the disconnected data shown in Fig. 3, we do not find large deviations from linearity. The quoted error is comparable to the change, $\approx 0.02$, between the lowest $M_{\pi}$ and $a$ point and the extrapolated value (red star), and thus a conservative estimate of possible residual uncertainty. Another estimate of the same systematic, $a^{2} \Lambda_{\mathrm{QCD}}^{2} \approx 0.02$, gives a similar value. We do include 0.02 , as an additional systematic for the sum of the disconnected contributions; combine it in quadrature with that from the connected contribution; and quote an overall second error of 0.036 in $\Delta \Sigma / 2=0.143(31)(36)$. This represents the model uncertainty of the chiral-continuum ansatz, i.e., using the lowest order corrections and fitting to a limited number of data points.

\section{COMPARISON WITH PREVIOUS WORK AND CONCLUSIONS}

In Fig. 4, we compare lattice results, restricted to publications including physical mass ensembles, with the moments extracted from global fits to the polarized PDFs reviewed in Ref. [4]. Within errors, our results are compatible with the moments extracted from global PDF fits, all expressed in the $\overline{\mathrm{MS}}$ scheme at $2 \mathrm{GeV}$. The ETMC lattice results from a single physical mass two-flavor ensemble at $a=0.093 \mathrm{fm}[17,27]$ are also consistent with ours. The small difference can be accounted for by the $a$ dependence highlighted in our disconnected contribution data shown in Fig. 3 , i.e., to get continuum limit values assuming similar
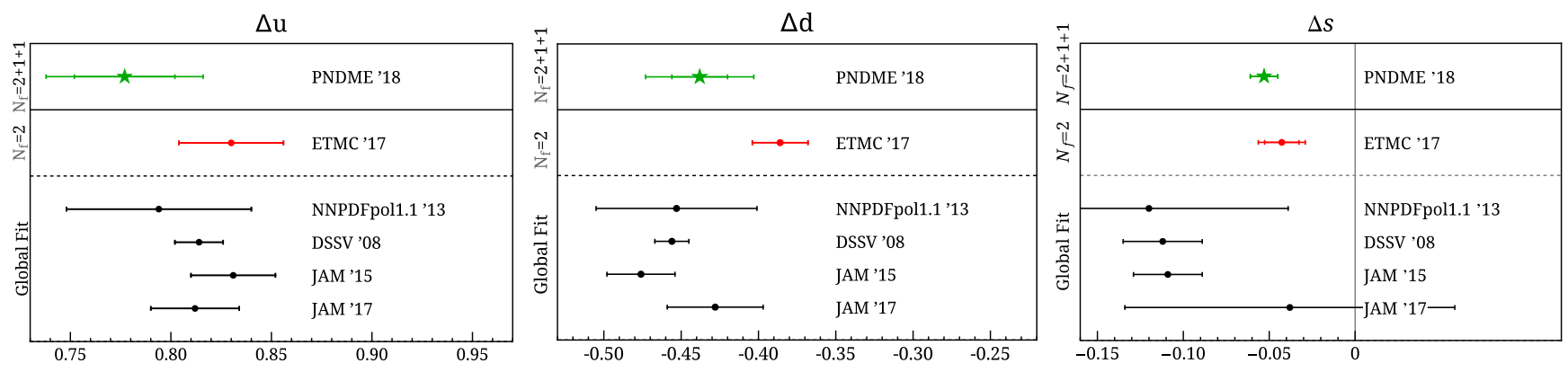

FIG. 4. Chiral-continuum extrapolated PNDME'18 results (this work) for $\Delta u, \Delta d$, and $\Delta s$ are compared with ETMC [17,27] values obtained at a single-lattice spacing and with moments from global fits to polarized PDF (NNPDFpol1.1'13 [28], DSSV'08 [29,30], Jam'15 [31], and JAM'17 [32]). All PDF results are taken from Ref. [4] and are at $2 \mathrm{GeV}$ in the $\overline{\mathrm{MS}}$ scheme. 
discretization errors, our fits indicate subtracting 0.04 from their $g_{A}^{u}$ and $g_{A}^{d}$ and 0.01 from $g_{A}^{s}$. The change in the connected contributions to $g_{A}^{u}$ and $g_{A}^{d}$ is only $O(0.01)$ [8]. Most likely, this is because the ETMC value for the isovector charge $g_{A}^{u-d}=1.212(40)$ is equally low. The difference in the two respective error estimates is mainly due to ETMC not including a systematic uncertainty to account for possible discretization effects in the connected and disconnected contributions, and therefore in $\Delta \Sigma$.

In conclusion, we present first results with chiralcontinuum extrapolation of up, down, and strange quark spin contributions. These fits are based on 6 (7) ensembles for the disconnected contribution of light (strange) quarks, and on 11 ensembles for the dominant connected contributions that were analyzed fully in Ref. [8]. We demonstrate in Fig. 3 that a chiral-continuum extrapolation significantly reduces the disconnected contribution of the quark spin to the proton spin and is, therefore, essential for getting physical results from lattice calculations. Our final result, $\frac{1}{2} \Delta \Sigma=0.143(31)(36)$, is consistent with the 2015 COMPASS analysis. Including more ensembles in the calculation of the disconnected contribution and resolving the $\approx 5 \%$ underestimate of $g_{A}^{u-d}$ that impacts the connected contributions are, at present, the largest systematics that need to be addressed in future works.

\section{ACKNOWLEDGMENTS}

We thank the MILC Collaboration for providing the $2+1+1$-flavor HISQ lattices. The calculations used the Chroma software suite [33]. Simulations were carried out on computer facilities at (i) the National Energy Research Scientific Computing Center, a DOE Office of Science User Facility supported by the Office of Science of the U.S. Department of Energy under Contract No. DE-AC02-05CH11231, and (ii) the Oak Ridge Leadership Computing Facility at the Oak Ridge National Laboratory, which is supported by the Office of Science of the U.S. Department of Energy under Contract No. DE-AC05-00OR22725. We also thank (iii) the USQCD Collaboration, which is funded by the Office of Science of the U.S. Department of Energy, (iv) Institutional Computing at Los Alamos National Laboratory, and (v) the Institute for Cyber-Enabled Research at Michigan State University. T. B. and R. G. were partly supported by the U.S. Department of Energy, Office of Science, Office of High Energy Physics under Contract No. DE-AC52-06NA25396. T. B., R. G., Y-C. J., and B. Y. were partly supported by the LANL LDRD program. The work of H.-W. L. is supported by the U.S. National Science Foundation under Grant No. PHY 1653405 "CAREER: Constraining Parton Distribution Functions for New-Physics Searches."
[1] J. Ashman et al. (European Muon Collaboration), Phys. Lett. B 206, 364 (1988).

[2] C. Adolph et al. (COMPASS Collaboration), Phys. Lett. B 753, 18 (2016).

[3] X.-D. Ji, Phys. Rev. Lett. 78, 610 (1997).

[4] H.-W. Lin et al., Prog. Part. Nucl. Phys. 100, 107 (2018).

[5] A. L. Fitzpatrick, W. Haxton, E. Katz, N. Lubbers, and Y. $\mathrm{Xu}, \operatorname{arXiv}: 1211.2818$.

[6] R. J. Hill and M. P. Solon, Phys. Rev. D 91, 043505 (2015).

[7] T. Bhattacharya, V. Cirigliano, S. Cohen, R. Gupta, A. Joseph, H.-W. Lin, and B. Yoon (PNDME Collaboration), Phys. Rev. D 92, 094511 (2015).

[8] R. Gupta, Y.-C. Jang, B. Yoon, H.-W. Lin, V. Cirigliano, and T. Bhattacharya, Phys. Rev. D 98, 034503 (2018).

[9] E. Follana, Q. Mason, C. Davies, K. Hornbostel, G. P. Lepage, J. Shigemitsu, H. Trottier, and K. Wong (HPQCD and UKQCD Collaborations), Phys. Rev. D 75, 054502 (2007).

[10] A. Bazavov et al. (MILC Collaboration), Phys. Rev. D 87, 054505 (2013).

[11] G. S. Bali, S. Collins, and A. Schafer, Comput. Phys. Commun. 181, 1570 (2010).

[12] T. Blum, T. Izubuchi, and E. Shintani, Phys. Rev. D 88, 094503 (2013).

[13] S. Sharpe (private communication).
[14] T. Bhattacharya, R. Gupta, W. Lee, S. R. Sharpe, and J. M. S. Wu, Phys. Rev. D 73, 034504 (2006).

[15] M. Constantinou, M. Hadjiantonis, H. Panagopoulos, and G. Spanoudes, Phys. Rev. D 94, 114513 (2016).

[16] C. Alexandrou et al., Phys. Rev. D 95, 114514 (2017); 96, 099906(E) (2017).

[17] C. Alexandrou, M. Constantinou, K. Hadjiyiannakou, K. Jansen, C. Kallidonis, G. Koutsou, A. Vaquero Avils-Casco, and C. Wiese, Phys. Rev. Lett. 119, 142002 (2017).

[18] J. Green, N. Hasan, S. Meinel, M. Engelhardt, S. Krieg, J. Laeuchli, J. Negele, K. Orginos, A. Pochinsky, and S. Syritsyn, Phys. Rev. D 95, 114502 (2017).

[19] V. Bernard, N. Kaiser, J. Kambor, and U. G. Meissner, Nucl. Phys. B388, 315 (1992).

[20] V. Bernard, N. Kaiser, and U.-G. Meissner, Int. J. Mod. Phys. E 04, 193 (1995).

[21] V. Bernard and U.-G. Meissner, Annu. Rev. Nucl. Part. Sci. 57, 33 (2007).

[22] V. Bernard and U.-G. Meissner, Phys. Lett. B 639, 278 (2006).

[23] A. A. Khan et al. (QCDSF Collaboration), Phys. Rev. D 74, 094508 (2006).

[24] G. Colangelo, A. Fuhrer, and S. Lanz, Phys. Rev. D 82, 034506 (2010).

[25] J. de Vries, R. Timmermans, E. Mereghetti, and U. van Kolck, Phys. Lett. B 695, 268 (2011). 
[26] H. Akaike, IEEE Trans. Autom. Control 19, 716 (1974).

[27] C. Alexandrou, M. Constantinou, K. Hadjiyiannakou, K. Jansen, C. Kallidonis, G. Koutsou, and A. V. Avils-Casco, EPJ Web Conf. 175, 06003 (2018).

[28] E. R. Nocera, R. D. Ball, S. Forte, G. Ridolfi, and J. Rojo (NNPDF Collaboration), Nucl. Phys. B887, 276 (2014).

[29] D. de Florian, R. Sassot, M. Stratmann, and W. Vogelsang, Phys. Rev. Lett. 101, 072001 (2008).
[30] D. de Florian, R. Sassot, M. Stratmann, and W. Vogelsang, Phys. Rev. D 80, 034030 (2009).

[31] N. Sato, W. Melnitchouk, S. E. Kuhn, J. J. Ethier, and A. Accardi (Jefferson Lab Angular Momentum), Phys. Rev. D 93, 074005 (2016).

[32] J. J. Ethier, N. Sato, and W. Melnitchouk, Phys. Rev. Lett. 119, 132001 (2017).

[33] R. G. Edwards and B. Joo (SciDAC, LHPC, and UKQCD Collaborations), Nucl. Phys. B, Proc. Suppl. 140, 832 (2005). 